\title{
Unidirectional flow of a thin rivulet on a vertical substrate subject to a prescribed uniform shear stress at its free surface
}

\author{
S. K. Wilson ${ }^{\text {a) }}$ and B. R. Duffy ${ }^{\text {b) }}$ \\ Department of Mathematics, University of Strathclyde, Livingstone Tower, 26 Richmond Street, \\ Glasgow G1 1XH, United Kingdom
}

(Received 8 October 2004; accepted 18 August 2005; published online 17 October 2005)

\begin{abstract}
We use the lubrication approximation to analyze the steady unidirectional flow of a thin rivulet on a vertical substrate subject to a prescribed uniform longitudinal shear stress at its free surface. In particular, we determine the quasisteady stability of a rivulet of prescribed flux, and calculate when it is energetically favorable for a rivulet to split into two narrower rivulets. (C) 2005 American Institute of Physics. [DOI: 10.1063/1.2100987]
\end{abstract}

Rivulets of fluid subject to significant surface-shear forces occur in a variety of contexts, including the rivulets of rainwater and/or deicing fluid that form on the wings of aircraft, the rivulets of condensate that frequently occur within heat exchangers, and even the rivulets of rainwater that occur on the windscreen of a rapidly moving car on a rainy day. Various aspects of rivulet flow in the presence of significant surface-shear forces have been investigated by, for example, Mikielewicz and Moszynski, ${ }^{1}$ Eres, Schwartz, and Roy, ${ }^{2}$ Wilson, Duffy, and Hunt, ${ }^{3}$ Myers, Liang, and Wetton, ${ }^{4}$ and Saber and El-Genk. ${ }^{5}$ In this Brief Communication we use the lubrication approximation to analyze the steady unidirectional flow of a thin rivulet on a vertical substrate subject to a prescribed uniform longitudinal shear stress at its free surface.

Consider the steady unidirectional flow of a thin symmetric rivulet with constant semiwidth $a$ and constant volume flux $Q$ on a vertical substrate subject to a prescribed uniform longitudinal shear stress $\tau$ at its free surface. We assume that the fluid is Newtonian and has constant density $\rho$, viscosity $\mu$, and surface tension $\gamma$. We choose Cartesian axes $O x y z$ with the $x$ axis vertically downwards and the $z$ axis normal to the substrate $z=0$. The velocity $\mathbf{u}=u(y, z) \mathbf{i}$ and pressure $p=p(x, y, z)$ of the fluid are governed by the familiar mass-conservation and Navier-Stokes equations subject to the usual normal and tangential stress balances and the kinematic condition at the (unknown) free surface $z=h(y)$, and zero velocity at the substrate $z=0$. At the contact line $y=a$, where $h=0$, the contact angle takes the prescribed value $\beta$, where $\beta>0$ is the (nonzero) static contact angle.

Analytical progress can be made when the rivulet is thin (with, in particular $\beta \ll 1$ ) in which case it is appropriate to nondimensionalize $y$ and $a$ with $l, z$ and $h$ with $\beta l, u$ with $U=\rho g \beta^{2} l^{2} / \mu, Q$ with $\beta l^{2} U=\rho g \beta^{3} l^{4} / \mu, p-p_{\infty}$ with $\rho g \beta l$, and $\tau$ with $\rho g \beta l$, where $l=(\gamma / \rho g)^{1 / 2}$ is the capillary length, $g$ is gravitational acceleration, and $p_{\infty}$ is atmospheric pressure.

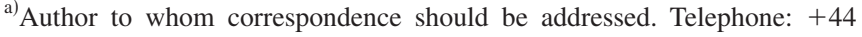
(0)141 548 3820. Fax: +44 (0)141 548 3345. Electronic mail: s.k.wilson@strath.ac.uk

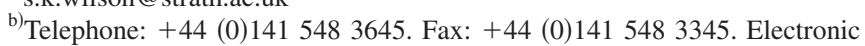
mail: b.r.duffy@strath.ac.uk
}

Henceforth all quantities are nondimensional unless it is stated otherwise. The leading-order problem can be immediately solved to yield the simple solution $p=-h^{\prime \prime}=1 / a, u$ $=(2 h-z) z / 2+\tau z, h=\left(a^{2}-y^{2}\right) / 2 a$. Note that since the flow is unidirectional, the mass-conservation equation and the kinematic condition are satisfied identically, and the solution is valid for all values of the Reynolds number. (In particular, the comment made by Myers et al. ${ }^{4}$ about the restriction on the applicability of the thin-film approximation is erroneous.) The maximum thickness of the rivulet occurs at $y=0$ and is equal to $h_{m}=h(0)=a / 2$. The flux $Q$ is given by

$$
Q=\int_{-a}^{+a} \int_{0}^{h(y)} u(y, z) \mathrm{d} z \mathrm{~d} y=\frac{4 a^{4}}{105}+\frac{2 \tau a^{3}}{15} .
$$

For $\tau \geqslant 0$ the flux $Q$ is a monotonically increasing function of $a$, but for $\tau<0$ the flux $Q$ initially decreases monotonically to a minimum value of $Q=Q_{\min }=-4 a_{\min }^{4} / 315$ $=-3087 \tau^{4} / 5120(<0)$ at $a=a_{\min }=-21 \tau / 8$, before increasing monotonically through the value $Q=0$ at $a=a_{0}=-7 \tau / 2$.

There are five possible types of flow pattern (denoted as types I-V) as sketched in Fig. 1. When $\tau>0$ the prescribed shear stress acts in the same direction as gravity. In this case the velocity is always downwards (i.e., $u>0$ ) throughout the rivulet, and the maximum velocity $u_{\max }=a(a+4 \tau) / 8(>0)$ occurs on the free surface at $y=0$ and $z=h_{m}=a / 2$ (type I). On the other hand, when $\tau<0$ the prescribed shear stress opposes gravity, and this competition leads to more interesting behavior than in the case $\tau>0$. In particular, the velocity is always upwards (i.e., $u<0$ ) near the edges of the rivulet, but it can be downwards (i.e., $u>0$ ) elsewhere. When $a \leqslant-2 \tau$ the velocity is always upwards throughout the rivulet, and the minimum velocity $u_{\min }=a(a+4 \tau) / 8(<0)$ occurs on the free surface at $y=0$ and $z=h_{m}=a / 2$ (type V), but when $a>-2 \tau$ there is a region of downwards flow in the center of the rivulet and the maximum velocity $u_{\max }=(a$ $+2 \tau)^{2} / 8(>0)$ occurs within the flow at $y=0$ and $z=h_{m}+\tau$ $=(a+2 \tau) / 2$, and the minimum velocity $u_{\min }=-\tau^{2} / 2(<0)$ occurs on the free surface at $y= \pm b= \pm a(1+2 \tau / a)^{1 / 2}$ and $z=-\tau$ (type II when $a>-4 \tau$, type III when $a=-4 \tau$ and type IV when $-2 \tau<a<-4 \tau)$. Note that both the solution $a=a_{0}$ $=-7 \tau / 2$ corresponding to $Q=0$ and the solution 
(a)

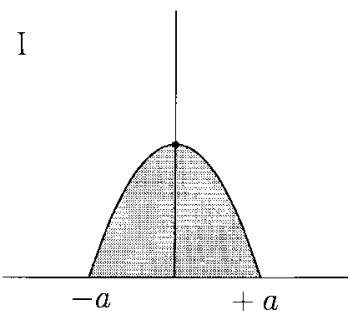

(c)

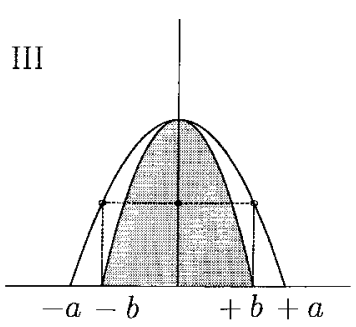

(e)

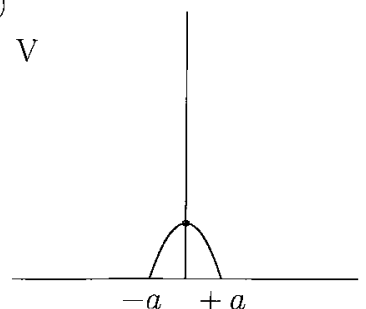

(b)

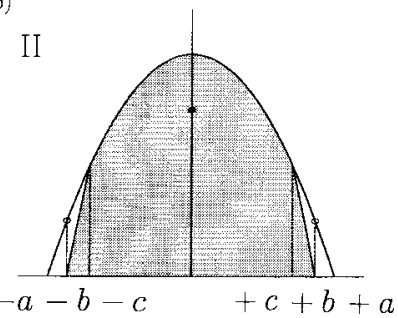

(d)

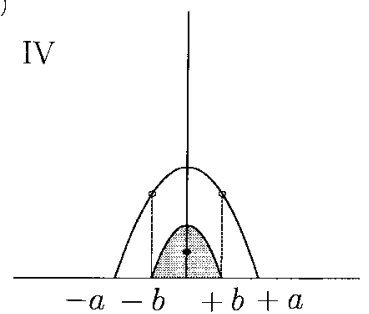

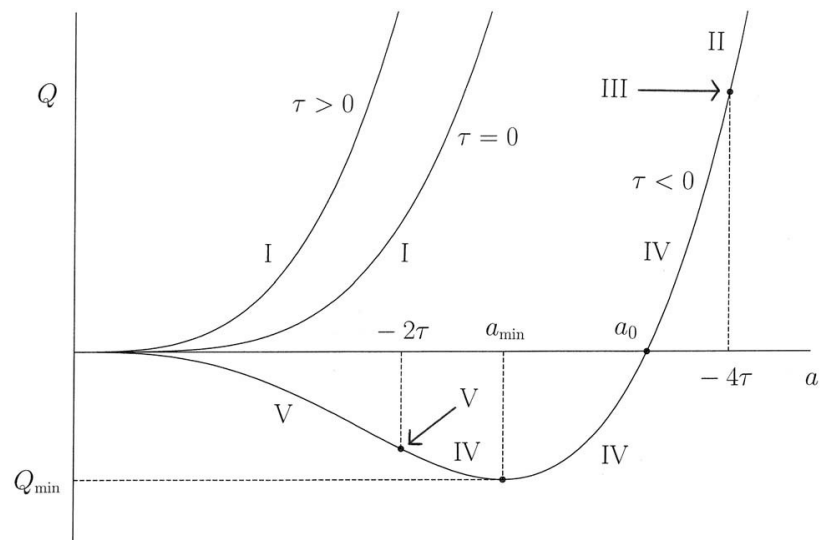

FIG. 2. Sketch of $Q$ as a function of $a$ summarizing when the different types of flow pattern occur for $\tau>0, \tau=0$, and $\tau<0$.

3 shows a sketch of $a$ as a function of $\tau$ and summarizes when the different types of flow pattern occur. The shape of the rivulet depends on $\tau$ via $a$. When $\tau>0$ the velocity is always downwards and the effect of increasing the shear stress from zero is always to increase the local velocity of the fluid throughout the rivulet, i.e., $\partial u / \partial \tau>0$ everywhere. Hence, since the flux must remain constant, the rivulet always becomes narrower (and shallower), i.e., $\partial a / \partial \tau$ $=-7 a /(8 a+21 \tau)<0$. When $\tau<0$ the velocity is always upwards near the edges of the rivulet, but may be downwards elsewhere. Moreover, the effect of decreasing the shear stress from zero can be either to increase or to decrease the local velocity. However, the net effect of the changes to the size of the rivulet and to the velocity along it are that when $a$ $<a_{\min }$ the rivulet always becomes narrower (and shallower), i.e., $\partial a / \partial \tau>0$, whereas when $a>a_{\min }$ the rivulet always becomes wider (and deeper), i.e., $\partial a / \partial \tau<0$.

A full stability analysis is beyond the scope of the present work, but in the case of prescribed flux $Q=\bar{Q}$ we can generalize the quasisteady stability analysis of a purely

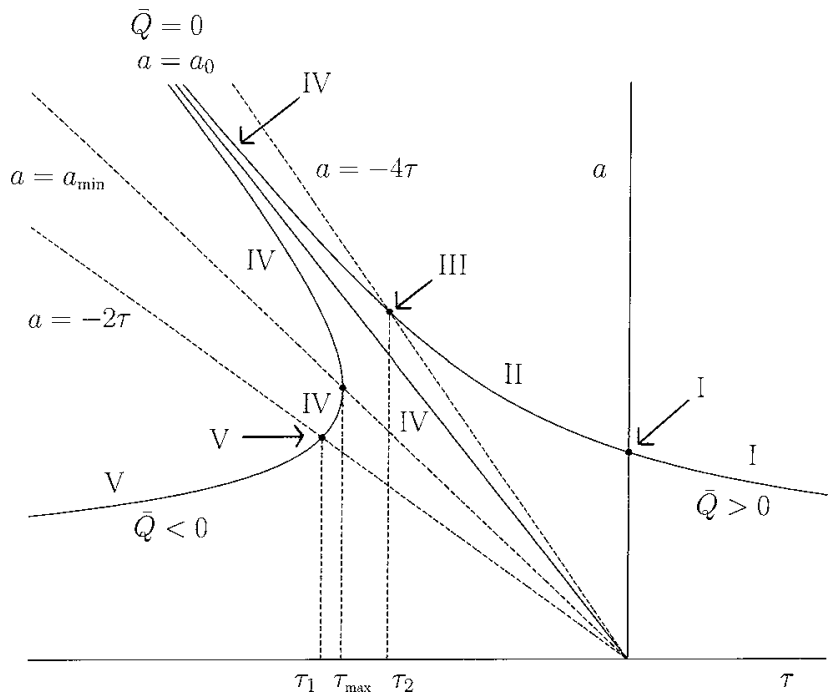

FIG. 3. Sketch of $a$ as a function of $\tau$ summarizing when the different types of flow pattern occur for $\bar{Q}>0, \bar{Q}=0$, and $\bar{Q}<0$. Here $\tau_{1}=-(-35 \bar{Q} / 16)^{1 / 4}$, $\tau_{\max }=-(-5120 \bar{Q} / 3087)^{1 / 4}$, and $\tau_{2}=-(105 \bar{Q} / 128)^{1 / 4}$. 
gravity-driven rivulet undertaken by Wilson and Duffy. ${ }^{6}$ Following the earlier work we assume that the flow remains symmetric and unidirectional and that the quasisteady motion is driven entirely by that of the moving contact line $y$ $=A$, where $A=A(t)$. Furthermore, we assume that the speed of the moving contact line, $A^{\prime}$, and the dynamic contact angle, $\theta=\theta(t)$, are related by a general "Tanner law" in the form $A^{\prime}=F(\theta)$, where the function $F(\theta)$ satisfies $F(1)=0$ and is monotonically increasing near $\theta=1$. Perturbing about the constant steady-state values of the semiwidth and the contact angle $A=a$ and $\theta=1$ by writing $A=a+a_{1}(t)$ and $\theta=1+\theta_{1}(t)$ yields

$$
a_{1}^{\prime}=\frac{M\left(\lambda a_{1}\right)^{m}}{m !}
$$

where $M=\mathrm{d}^{m} F /\left.\mathrm{d} \theta^{m}\right|_{\theta=1}>0(m=1,3,5, \ldots)$ is the first nonzero derivative of $F(\theta)$ evaluated at $\theta=1$ and

$$
\lambda=-\left.\frac{Q_{A}}{Q_{\theta}}\right|_{A=a, \theta=1}=-\frac{8 a+21 \tau}{2 a(3 a+7 \tau)} .
$$

Equation (2) can be immediately solved for $a_{1}$ and this solution shows that, whatever the specific form of $F(\theta)$, the rivulet is unstable to small perturbations when $\lambda>0$ and stable when $\lambda<0$. Hence when $\tau \geqslant 0$ and when $\tau<0$ and $\bar{Q} \geqslant 0$ the rivulet is always stable, while when $\tau<0$ and $Q_{\min } \leqslant \bar{Q}<0$ the wider rivulet is always stable and the narrower rivulet is stable when $0<a<-7 \tau / 3$ but unstable when $-7 \tau / 3<a<a_{\min }=-21 \tau / 8$.

We can also address the question posed by Schmuki and Laso $^{7}$ and Myers et al. ${ }^{4}$ of whether or not it is ever energetically favorable for a rivulet to split into two or more subrivulets. The total energy of the rivulet is the sum of its kinetic energy and surface energy. Momentarily reverting to dimensional variables, the kinetic energy (per unit length) is given by

$$
\frac{\rho}{2} \int_{-a}^{+a} \int_{0}^{h} u^{2} \mathrm{~d} z \mathrm{~d} y
$$

and the surface energy, or, more precisely, the difference between the surface energy of the rivulet and the surface energy of the dry substrate (per unit length) is given by

$$
\gamma\left[\int_{-a}^{+a}\left(1+h^{\prime 2}\right)^{1 / 2} \mathrm{~d} y-2 a \cos \beta\right] .
$$

Thus, if we nondimensionalize energy (per unit length) with $\rho U^{2} \beta l^{2}=\rho^{3} g^{2} \beta^{5} l^{6} / \mu^{2}$ then the leading-order energy of the rivulet, $E$, is given by

$$
E=\int_{-a}^{+a} \frac{h^{5}}{15}+\frac{5 \tau h^{4}}{24}+\frac{\tau^{2} h^{3}}{6} \mathrm{~d} y+\frac{1}{W}\left[\frac{1}{2} \int_{-a}^{+a} h^{\prime 2} \mathrm{~d} y+a\right],
$$

which can be evaluated explicitly to yield

$$
E=\frac{16 a^{6}}{10395}+\frac{2 \tau a^{5}}{189}+\frac{2 \tau^{2} a^{4}}{105}+\frac{4 a}{3 W},
$$

where $W=\rho l U^{2} / \gamma \beta=\gamma^{2} \beta^{3} / g l \mu^{2}$ is an appropriately defined Weber number. ${ }^{8}$ It can readily be demonstrated that (for a fixed value of $\tau$ ) $E$ is a monotonically increasing function of $a$. Thus wider rivulets always have more energy than narrower ones, and hence it is never energetically favorable for a rivulet to split into one or more wider rivulets. However, it can be energetically favorable for a rivulet to split into one or more narrower rivulets. Specifically, it is energetically favorable for a rivulet with semiwidth $a$ and flux $Q$ to split into two rivulets, one with flux $\lambda Q$ and the other with flux (1 $-\lambda) Q$, where $0<\lambda \leqslant 1 / 2$, if the difference between the energies of the two states, $\Delta E$, is positive.

In the special case of a purely gravity-driven rivulet (i.e., the case $\tau=0)$ the semiwidth is given by $a=(105 Q / 4)^{1 / 4}$, and when $a<a_{c}$ then $\Delta E<0$ for all $0<\lambda \leqslant 1 / 2$, but when $a$ $=a_{c}$ then $\Delta E=0$ at $\lambda=1 / 2$, and when $a>a_{c}$ then $\Delta E>0$ at $\lambda=1 / 2$. Thus for $a>a_{c}$ (or equivalently for $Q>Q_{c}$ ) it is energetically favorable for the rivulet to split into two equal narrower rivulets, where the critical values of the semiwidth, $a_{c}$, and the flux, $Q_{c}$, are given by

$$
\begin{aligned}
& a_{c}=\left[\frac{3465\left(2^{3 / 4}-1\right)}{4\left(1-2^{-1 / 2}\right) W}\right]^{1 / 5} \simeq \frac{4.5805}{W^{1 / 5}}, \\
& Q_{c}=\frac{4}{105}\left[\frac{3465\left(2^{3 / 4}-1\right)}{4\left(1-2^{-1 / 2}\right) W}\right]^{4 / 5} \simeq \frac{16.7703}{W^{4 / 5}} .
\end{aligned}
$$

This result agrees qualitatively with Myers et al., ${ }^{4}$ who calculated $a_{c}$ and $Q_{c}$ numerically for a range of values of $\beta$.

In the limit of large positive shear stress $\tau \rightarrow \infty$ when $Q>0$, and for the narrower rivulet in the limit of large negative shear stress $\tau \rightarrow-\infty$ when $Q<0$, the leading order semiwidth is given by $a=(15 Q / 2 \tau)^{1 / 3}$, and the behavior of $\Delta E$ is qualitatively the same as that in the case $\tau=0$ described above, where $a_{c}$ and $Q_{c}$ are now given by

$$
\begin{aligned}
& a_{c}=\left[\frac{70\left(2^{2 / 3}-1\right)}{\left(1-2^{-1 / 3}\right) \tau^{2} W}\right]^{1 / 3} \simeq \frac{5.8413}{\tau^{2 / 3} W^{1 / 3}}, \\
& Q_{c}=\frac{28\left(2^{2 / 3}-1\right)}{3\left(1-2^{-1 / 3}\right) \tau W} \simeq \frac{26.5750}{\tau W} .
\end{aligned}
$$

In particular, this result shows that the conjecture proposed by Myers et al. ${ }^{4}$ that it is never energetically favorable for a purely shear-stress-driven rivulet to split is not correct.

When both gravity and shear-stress effects are significant analytical progress is harder. However, we can still make progress numerically. Before doing this it is convenient to remove $W$ from the problem by scaling $a$ with $W^{-1 / 5}, \tau$ with $W^{-1 / 5}, Q$ with $W^{-4 / 5}$, and $E$ with $W^{-6 / 5}$. When $Q>0$ the behavior is qualitatively the same as that in the case $\tau=0$. When $Q_{\min } \leqslant Q<0$ (which is possible only when $\tau<0$ ) the situation is somewhat more complicated. In this case there are always two possible rivulets with the same flux (the narrower one satisfying $0<a \leqslant a_{\min }$ and the wider one $a_{\min }$ $\left.<a<a_{0}\right)$. As we have already seen, it is always energetically favorable for the wider rivulet to "split" into the narrower 


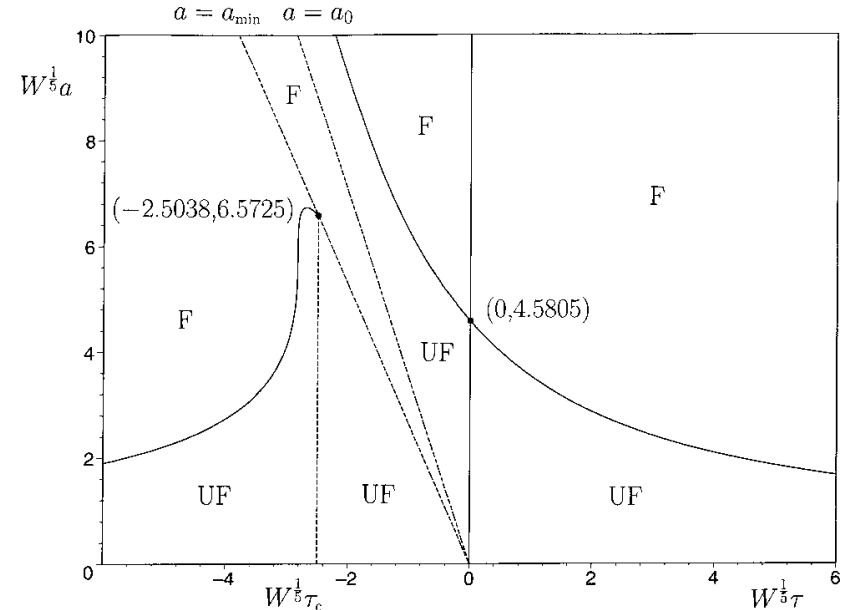

FIG. 4. Plot of $W^{1 / 5} a$ as a function of $W^{1 / 5} \tau$ indicating when it is energetically favorable (denoted by "F") or unfavorable (denoted by "UF") for a rivulet to split. The curves $a=a_{\min }=-21 \tau / 8$ and $a=a_{0}=-7 \tau / 2$ are indicated with dashed lines.

rivulet with the same flux. On the other hand, the behavior of the narrower rivulet is somewhat similar to that of the rivulets in the case $Q>0$ described previously. Specifically, for $\tau_{c} \leqslant \tau<0$, where $\tau_{c} \simeq-2.5038 \mathrm{~W}^{-1 / 5}$, it is never energetically favorable for the smaller rivulet to split, but for $\tau<\tau_{c}$ there is a critical value of the semiwidth $a_{c}\left(0<a_{c} \leqslant a_{\min }\right)$ above which and a critical value of the flux $Q_{c}\left(Q_{\min } \leqslant Q_{c}<0\right)$ below which it is energetically favorable for the rivulet to split into two narrower rivulets, and the critical situation is again that of splitting into two equal rivulets each with half the flux of the original, i.e., the case $\lambda=1 / 2$. Note that $a_{c}=a_{\min }\left(\tau_{c}\right) \simeq 6.5725 W^{-1 / 5}$ and $Q_{c}=Q_{\min }\left(\tau_{c}\right)$ $\simeq-23.6954 W^{-4 / 5}$. Figures 4 and 5 show $W^{1 / 5} a$ and $W^{4 / 5} Q$, respectively, plotted as functions of $W^{1 / 5} \tau$ and indicate when it is energetically favorable or unfavorable for a rivulet to split.

Note that, although perhaps slightly obscured by the choice of nondimensionalization, the results described in the present work include the very simple special case of a purely shear-stress-driven rivulet. Specifically, if we revert to dimensional variables and set $g=0$ then we have $u=\tau z / \mu$ and $Q=2 \tau \beta^{2} a^{3} / 15 \mu$. In this case there is always single rivulet for each value of $\bar{a}(>0)$ or $\bar{Q}$ (provided that it has the same

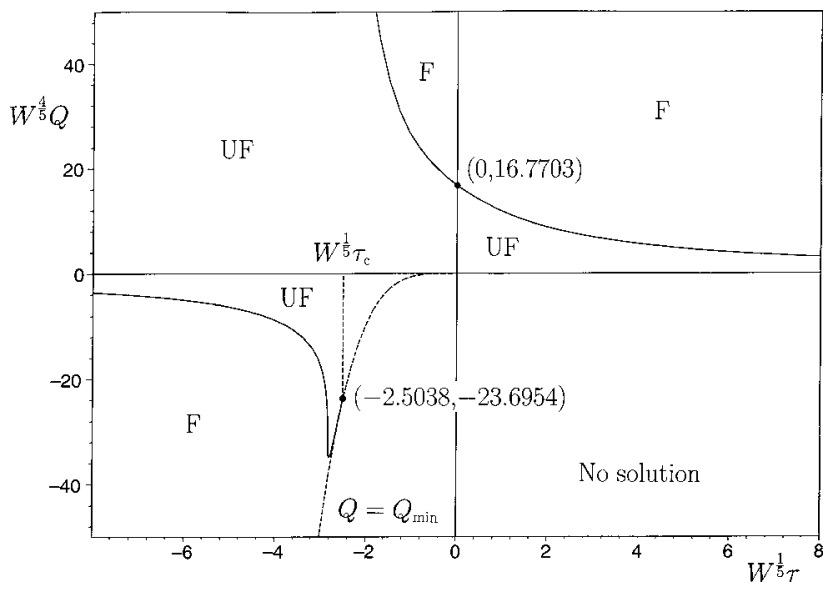

FIG. 5. Plot of $W^{4 / 5} Q$ as a function of $W^{1 / 5} \tau$ indicating when it is energetically favorable (denoted by "F") or unfavorable (denoted by "UF") for a rivulet to split. The curve $Q=Q_{\min }=-3087 \tau^{4} / 5120$ in $\tau \leqslant 0$ is indicated with a dashed line. Note that when $\tau<0$ and $Q_{\min }<Q<0$ the region in which it is unfavorable to split applies only to the narrower rivulet.

sign as $\tau$ ), and the flow is always in the same direction as the prescribed shear stress throughout the rivulet (i.e., the flow pattern is always of type I when $\tau>0$ and always of type $\mathrm{V}$ when $\tau<0$ ).

${ }^{1}$ J. Mikielewicz and J. R. Moszynski, "An improved analysis of breakdown of thin liquid films," Arch. Mech. 30, 489 (1978).

${ }^{2}$ M. H. Eres, L. W. Schwartz, and R. V. Roy, "Fingering phenomena for driven coating films," Phys. Fluids 12, 1278 (2000).

${ }^{3}$ S. K. Wilson, B. R. Duffy, and R. Hunt, "A slender rivulet of a power-law fluid driven by either gravity or a constant shear stress at the free surface," Q. J. Mech. Appl. Math. 55, 385 (2002).

${ }^{4}$ T. G. Myers, H. X. Liang, and B. Wetton, "The stability and flow of a rivulet driven by interfacial shear and gravity," Int. J. Non-Linear Mech. 39, 1239 (2004).

${ }^{5}$ H. H. Saber and M. S. El-Genk, "On the breakup of a thin liquid film subject to interfacial shear," J. Fluid Mech. 500, 113 (2004).

${ }^{6} \mathrm{~S}$. K. Wilson and B. R. Duffy, "On the gravity-driven draining of a rivulet of viscous fluid down a slowly varying substrate with variation transverse to the direction of flow," Phys. Fluids 10, 13 (1998).

${ }^{7}$ P. Schmuki and M. Laso, "On the stability of rivulet flow," J. Fluid Mech. 215, 125 (1990).

${ }^{8}$ The expression for the kinetic energy in (7) is in agreement with the appropriate limit of the corresponding result given by Myers et al. [see Ref. 4, Eq. (40)] if the typographical error in their coefficient $a_{2}$ is corrected (specifically, their $m L$ should be $\sqrt{C}$ ). The expression for the surface energy in (7) corrects the corresponding result given by Myers et al. [see Ref. 4, Eq. (41)], who mistakenly omitted a contribution due to the surface energy of the free surface. 